\title{
The Impact of Perioperative Factors on Changes in Diastolic Function after Kidney Transplantation: A Retrospective Analysis
}

\author{
Eun Jung Kim ${ }^{1,2}$, Bon-Nyeo Koo ${ }^{1,2}$, So Yeon Kim ${ }^{1,2}$, Kyu Ha Huh ${ }^{3}$, Soojeong Kang ${ }^{1}$, and Yong Seon Choi ${ }^{1,2}$ \\ Departments of ${ }^{1}$ Anesthesiology and Pain Medicine and ${ }^{3}$ Transplantation Surgery, Severance Hospital, Yonsei University College of Medicine, \\ Seoul; \\ ${ }^{2}$ Anesthesia and Pain Research Institute, Yonsei University College of Medicine, Seoul, Korea.
}

Purpose: Cardiac changes in end-stage renal disease are the most common causes of death after kidney transplantation (KT). Chronic kidney disease presents a major risk factor for the development and progression of diastolic dysfunction. The purpose of this study was to identify the association between changes in left ventricular (LV) diastolic function and perioperative clinical factors in patients with preserved ejection fraction following KT.

Materials and Methods: We reviewed 115 patients who underwent KT between January 2011 and December 2015 with both preand post-transplant echocardiograms; patients with LV systolic dysfunction were excluded. LV diastolic function was measured using the ratio of early transmitral flow velocity to early diastolic velocity of the mitral annulus (E/e').

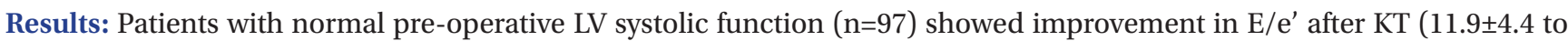
$10.5 \pm 3.8, p=0.023$ ). Additionally, post-KT estimated glomerular filtration ratio was associated with changes in $\mathrm{E} / \mathrm{e}^{\prime}$ (odds ratio, $-0.056 ; 95 \%$ confidence interval, -0.014 to $-0.007 ; p=0.026$ ). Among patients with preexisting diastolic dysfunction ( $20 / 97$ patients), the amount of intraoperative fluid administration was related to $\mathrm{E} / \mathrm{e}^{\prime}$ changes (odds ratio, $0.003 ; 95 \%$ confidence interval, 0.000 to $0.005 ; p=0.029$ ).

Conclusion: KT is associated with improved diastolic function. Post-KT renal function was significantly related to changes in LV diastolic function. The amount of intraoperative fluid was a risk factor for worsening diastolic function after KT in patients with preexisting diastolic dysfunction.

Key Words: Kidney transplantation, end-stage renal disease (ESRD), diastolic function

\section{INTRODUCTION}

Patients with end-stage renal disease (ESRD) are accompanied by hypertension, increased volume, hyperparathyroid-

Received: July 16, 2018 Revised: December 3, 2018

Accepted: January 3, 2019

Corresponding author: Yong Seon Choi, MD, PhD, Department of Anesthesiology and Pain Medicine, Anesthesia and Pain Research Institute, Yonsei University College of Medicine, 50-1 Yonsei-ro, Seodaemun-gu, Seoul 03722, Korea.

Tel: 82-2-2228-2412, Fax: 82-2-2227-7897, E-mail: YSCHOI@yuhs.ac

-The authors have no potential conflicts of interest to disclose.

(C) Copyright: Yonsei University College of Medicine 2019

This is an Open Access article distributed under the terms of the Creative Commons Attribution Non-Commercial License (https://creativecommons.org/licenses/ by-nc/4.0) which permits unrestricted non-commercial use, distribution, and reproduction in any medium, provided the original work is properly cited. ism, ${ }^{1,2}$ and even uremia, all of which result in higher left ventricular (LV) filling pressure, abnormal myocardial relaxation, thickened LV due to fibrosis, ${ }^{3}$ and ultimately diastolic dysfunction. Diastolic dysfunction, assessed using echocardiography, is associated with a marked increase in all-cause mortality in population-based studies, especially in patients with ESRD, because these patients are exposed to hemodynamic overload from excessive volume and pressure, anemia, arteriovenous shunts, and arterial remodeling. ${ }^{3-5}$

Renal transplantation is the treatment of choice for ESRD. Its impact on restoration of organ function makes it a promising treatment modality for patients with ESRD, not only for the renal system, but also for the cardiovascular system. Previous studies evaluating the effect of renal transplantation on the cardiovascular system have shown the resolution of LV hy- 
pertrophy and systolic dysfunction after transplantation; however, the impact on diastolic dysfunction was less significant, as dysfunction may persist or even worsen after transplantation. ${ }^{5-8}$ Since patients with ESRD undergoing renal transplantation are subject to hemodynamic changes during the perioperative period, proper perioperative management in relation to diastolic function needs to be emphasized. Comprehensive perioperative management, accounting for changes in such parameters, would assist with an ideal management strategy for groups of patients who are vulnerable to diastolic dysfunction, such as patients with ESRD undergoing kidney transplantation (KT).

The ratio of LV transmitral early peak velocity to early diastolic mitral annulus velocity (E/e'), measured using transthoracic echocardiography (TTE), is the most reliable non-invasive predictor of elevated LV filling pressure in ESRD. ${ }^{9}$ Moreover, E/e' ratio is a strong predictor of major adverse cardiovascular events, other than ejection fraction (EF), in patients with ESRD. ${ }^{10,11}$ Previous studies have shown that perioperative conditions may be associated with cardiac alterations after renal transplantation due to dynamic changes in intravascular volume and metabolic imbalance., ${ }^{6,12}$ The purpose of this study was to determine the perioperative clinical and laboratory factors that may be associated with changes in diastolic function, in terms of E/e' ratio, among patients with ESRD receiving KT.

\section{MATERIALS AND METHODS}

\section{Study design}

This study was a single-center retrospective analysis of a consecutive cohort of adult patients with ESRD who underwent KT between January 2011 and December 2015 and had preand post-transplant TTEs. All transplant recipients were generally administered the standard immunosuppressive protocol, which included induction therapy (anti-interleukin-2 receptor antibody), calcineurin inhibitor, mycophenolate, and steroid. Tapering of steroids was performed with the intent to transition into a steroid-free regimen after the first year if no former rejection episodes had occurred. Patients without pre- and/or post-transplant TTE and those who did not have adequate medical records regarding surgery and anesthesia were excluded. In addition, patients with LV systolic dysfunction ( $\mathrm{EF}<52 \%$ for males and $<54 \%$ for females) were excluded. This study's procedures were approved by and conducted in accordance with the ethical standards of the Yonsei University Health System Institutional Review Board (IRB no. 4-2017-0104). The participant informed consent requirement was waived by the ethics committee due to the study's retrospective design.

\section{Data collection}

Demographic, clinical, echocardiographic, and laboratory data were obtained directly from patient electronic medical re- cords. Baseline patient demographic information, including the duration of ESRD and dialysis, types of dialysis, and underlying medical comorbidities, were obtained from individual patient interviews performed in the outpatient clinic prior to surgery. The underlying conditions related to renal failure (e.g., glomerulonephritis, diabetes, hypertension, and polycystic kidney disease) were attained from pathologic confirmation. Intraoperative hemodynamic variables, including heart rate and blood pressure, were collected from the electronic anesthetic records. Information on intraoperative fluid balance, including estimated blood loss, urine output, fluid consumption, red blood cell concentration, and fresh frozen plasma, was also collected from the anesthetic records. The amount of fluid administered, estimated blood loss, and urine output during the post-operative period were collected from the vital sign records in the electronic medical record. The estimated glomerular filtration rate (eGFR) was calculated using the Modification of Diet in Renal Disease equation. ${ }^{13}$ Anemia was defined as hemoglobin $(\mathrm{Hb})<13 \mathrm{~g} / \mathrm{dL}$ in males and $<12$ $\mathrm{g} / \mathrm{dL}$ in females, according to the Kidney Disease: Improving Global Outcomes (KDIGO) guidelines for patients with chronic kidney disease. ${ }^{14}$

\section{Echocardiography and Doppler measurements}

Pre-transplantation TTE was performed on the day after each patient's regular hemodialysis schedule, while the post-transplantation data were obtained from the TTE taken most recently after surgery. Echocardiography was performed by experienced physicians from the echocardiography laboratory of the Cardiology Department at Severance Hospital in South Korea. Comprehensive TTE was performed using commercially available equipment. Cardiac dimensions were acquired using M-Mode echocardiography or directly from two-dimensional images. Left ventricular ejection fraction (LVEF), an indicator of LV systolic function, was calculated using a modified biplane Simpson's method from the apical two-chamber and four-chamber views. Transmitral Doppler inflow at the tips of the mitral leaflets was measured to obtain E-wave velocity, E-wave deceleration time, A-wave velocity, and E/A ratio. Average peak early diastolic velocity ( $\mathrm{e}^{\prime}$ ) was obtained from the septal and lateral sides of the mitral annulus in the apical four-chamber view with proper pulsed-wave tissue Doppler imaging (TDI) settings. The ratio of LV early diastolic filling velocity to the peak diastolic velocity of mitral medial annulus (E/ $\mathrm{e}^{\prime}$ ), measured using TTE, was calculated and used as an index of LV diastolic function and LV filling pressures. ${ }^{10,15,16}$ Reference values for echocardiographic parameters were defined according to the American Society of Echocardiography and European Association of Cardiovascular Imaging guidelines ${ }^{17,18}$ as four recommended variables and their abnormal cut-off values as follows: annular e' velocity (septal $\mathrm{e}^{\prime}<7 \mathrm{~cm} / \mathrm{sec}$, lateral $\mathrm{e}^{\prime}<10 \mathrm{~cm} / \mathrm{sec}$ ); average E/e' ratio $>14$; left atrial (LA) maximum volume index $>34 \mathrm{~mL} / \mathrm{m}^{2}$; and peak tricuspid regurgi- 
tant velocity $>2.8 \mathrm{~m} / \mathrm{sec}$.

\section{Statistical analysis}

Continuous data are expressed as a mean \pm standard deviation or median (interquartile range), depending on the distribution of data, and categorical data are expressed as a number (\%). Accordingly, paired t-test or Wilcoxon test was used for comparisons of paired observations. Categorical variables were compared using chi-squared tests. Linear regression analysis was used to analyze associations between clinical variables and changes in E/e'. Statistical analyses were performed using SPSS v23.0 (IBM Corporation, Armonk, NY, USA) software; $p<$ 0.050 was considered statistically significant.

\section{RESULTS}

Of 516 adult patients who underwent KT between January 2011 and December 2015, we identified 115 patients who fulfilled the inclusion and exclusion criteria (Fig. 1). Eighteen patients were excluded from the study due to insufficient pre- or postoperative TTE data $(\mathrm{n}=10)$ and impaired baseline LV systolic function $(n=8)$, resulting in 97 patients that were analyzed in this study.

The baseline patient characteristics are shown in Table 1. The mean age was $49.5 \pm 10.6 \mathrm{y}$, and 71 (62\%) patients were male. The etiology of ESRD was not defined in most patients (36\%), followed by hypertension (26\%) and diabetes mellitus (15\%). The majority of the patients was dialysis-dependent: 94 (82\%) patients were treated with hemodialysis and 15 (13\%) patients with peritoneal dialysis. The median duration of dialysis was 4 months (1-24 months). Hypertensive and heart failure patients were taking a range of antihypertensive medications, including calcium channel blockers (63\%), beta-blockers $(47 \%)$, angiotensin-converting enzyme inhibitors or angiotensin II receptor blockers (58\%), and diuretics (26\%).

The changes in structural TTE parameters and clinical and

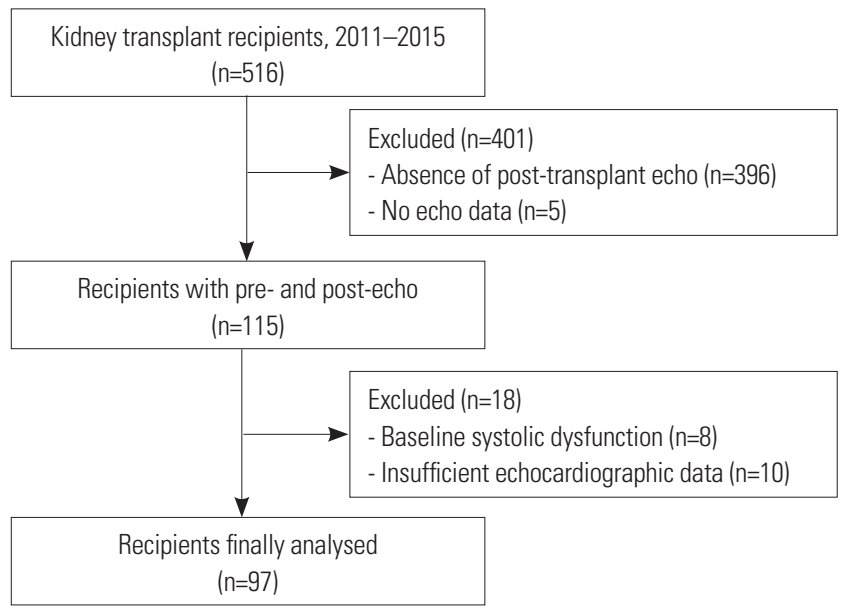

Fig. 1. Flow diagram of patients. laboratory variables in the post-transplantation period are shown in Table 2. Of note, none of the patients underwent coronary re-vascularization or cardiac re-synchronization therapy. Statistically significant improvements were observed in LVEF, LV mass/body surface area, LV end-diastolic diameter, LA volume index (LAVI), and E/e' ratio. The mean E/e' ratio significantly decreased after KT in patients with any degree of diastolic function ( $\mathrm{n}=97 ; p=0.023)$ and in those with underlying diastolic dysfunction $(n=20 ; p<0.001)$. The average time interval between KT and the follow-up TTE examination was 22.4 months, while the TTE had been done earlier in those with underlying diastolic dysfunction (average time interval of 18.9 months with earliest examination done at postoperative day 1).

The degrees of $\mathrm{Hb}$ increment after surgery were $1.1 \mathrm{~g} / \mathrm{dL}$ and $1.3 \mathrm{~g} / \mathrm{dL}(p<0.001$ for both) in all patients and in those with baseline anemia, respectively. Additionally, eGFR significantly improved during the post-operative follow-up period (Table 2).

Univariate analysis indicated that restoration of renal function, according to the increment of eGFR at post-operative day 7 and at 1 year post-operation, was significantly associat-

Table 1. Baseline Patient Characteristics

\begin{tabular}{lccc}
\hline & $\begin{array}{c}\text { Pre- and Post- } \\
\text { transplant TTE } \\
\text { (n=115) }\end{array}$ & $\begin{array}{c}\text { Pre-transplant } \\
\text { TTE (n=396) }\end{array}$ & $\begin{array}{c}\text { No TTE } \\
\text { (n=5) }\end{array}$ \\
\hline Transplant age (yr) & $49.5 \pm 10.6$ & $44.9 \pm 11.4^{*}$ & $49.2 \pm 6.9$ \\
Male & $71(62)$ & $195(49)$ & $3(60)$ \\
\hline Body mass index $\left(\mathrm{kg} / \mathrm{m}^{2}\right)$ & $23.2 \pm 3.1$ & $22.3 \pm 3.0^{*}$ & $21.1 \pm 1.8$ \\
Body surface area (m²) & $1.69 \pm 0.16$ & $1.67 \pm 0.18$ & $1.61 \pm 0.11$ \\
Renal condition & & & \\
\hline Diabetes mellitus & $17(15)$ & $12(3)^{*}$ & $2(40)^{\dagger . \ddagger}$ \\
\hline Hypertension & $30(26)$ & $82(21)$ & $1(20)$ \\
\hline Glomerulonephritis & $16(14)$ & $83(21)$ & 0 \\
\hline Polycystic kidney disease & $4(3)$ & $10(3)$ & $1(20)^{\ddagger}$ \\
\hline Others & $7(6)$ & $25(6)$ & 0 \\
\hline Not defined & $41(36)$ & $184(46)$ & $1(20)$ \\
\hline ESRD (yr) & $3.0(1.3-8.0)$ & $2.0(0-11.0)$ & $1.0(0.8-4.5)$ \\
Dialysis duration (month) & $4.0(1.0-24.0)$ & $3.0(0.5-7.0)^{*}$ & $1.0(0-1.0)^{\dagger}$ \\
Comorbidities & & & \\
\hline Diabetes mellitus & $37(32)$ & $86(22)^{*}$ & $4(80)^{\dagger, \ddagger}$ \\
\hline Hypertension & $101(88)$ & $245(62)$ & $3(60)$ \\
\hline CAOD & $18(16)$ & $9(2)^{*}$ & $1(20)^{\ddagger}$ \\
\hline COPD & $1(1)$ & $3(1)$ & 0 \\
\hline Hyperlipidemia & $20(17)$ & $38(10)$ & 0 \\
\hline Heart failure & $5(4)$ & $3(1)$ & 0 \\
\hline
\end{tabular}

$T \mathrm{TE}$, transthoracic echocardiography; ESRD, end-stage renal disease; CAOD, coronary artery obstructive disease; COPD, chronic obstructive pulmonary disease.

Data are presented as mean \pm standard deviation, median (interquartile range), or $n(\%)$.

${ }^{*} p<0.05$ between pre- and post-transplant TTE and pre-transplant TTE group, ${ }^{\dagger} p<0.05$ between pre- and post-transplant TTE and no TTE group, ${ }^{\ddagger} p<0.05$ between pre-transplant TTE group and no TTE group. 
Table 2. Post-Transplantation Outcomes

\begin{tabular}{|c|c|c|c|}
\hline Variable & Pre-transplant & Post-transplant & $p$ value \\
\hline $\operatorname{LVEF}(\%)$ & $66.4 \pm 5.8$ & $68.2 \pm 5.5$ & 0.037 \\
\hline \multicolumn{4}{|l|}{$\begin{array}{l}\text { LV mass/body surface area } \\
\left(\mathrm{g} / \mathrm{m}^{2}\right)\end{array}$} \\
\hline All patients & $129.4 \pm 34.6$ & $108.7 \pm 33.4$ & $<0.001$ \\
\hline $\begin{array}{l}\text { Baseline LV hypertrophy } \\
(\mathrm{n}=78)\end{array}$ & $138.6 \pm 31.6$ & $111.8 \pm 35.5$ & $<0.001$ \\
\hline \multicolumn{4}{|l|}{ LV end-diastolic diameter (cm) } \\
\hline All patients & $5.2 \pm 0.5$ & $4.8 \pm 0.4$ & $<0.001$ \\
\hline Baseline $\geq 5.6$ ( $n=18$ ) & $5.9 \pm 0.2$ & $5.0 \pm 0.5$ & $<0.001$ \\
\hline \multicolumn{4}{|l|}{$\mathrm{LA}$ volume index $\left(\mathrm{mL} / \mathrm{m}^{2}\right)$} \\
\hline All patients & $33.8 \pm 11.1$ & $29.2 \pm 8.5$ & 0.002 \\
\hline Baseline, mild $\geq 35(n=42)$ & $44.0 \pm 7.6$ & $33.2 \pm 8.7$ & $<0.001$ \\
\hline $\begin{array}{l}\text { Baseline, moderate } \geq 42 \\
\quad(n=22)\end{array}$ & $49.3 \pm 6.9$ & $34.7 \pm 8.7$ & $<0.001$ \\
\hline \multicolumn{4}{|l|}{ E/e' ratio } \\
\hline All patients & $11.9 \pm 4.4$ & $10.5 \pm 3.8$ & 0.023 \\
\hline $\begin{array}{l}\text { Diastolic dysfunction } \\
(\mathrm{n}=20)\end{array}$ & $18.4 \pm 3.7$ & $12.1 \pm 4.7$ & $<0.001$ \\
\hline \multicolumn{4}{|l|}{ RV systolic pressure $(\mathrm{mm} \mathrm{Hg})$} \\
\hline All patients & $27.8 \pm 9.3$ & $26.2 \pm 6.7$ & 0.191 \\
\hline Baseline $\geq 40(n=10)$ & $48.0 \pm 6.1$ & $29.1 \pm 7.7$ & $<0.001$ \\
\hline \multicolumn{4}{|l|}{$\begin{array}{l}\text { Mean blood pressure } \\
(\mathrm{mm} \mathrm{Hg})\end{array}$} \\
\hline All patients & $111.0 \pm 11.9$ & $107.8 \pm 15.7$ & 0.103 \\
\hline \multicolumn{4}{|l|}{ Hemoglobin (g/dL) } \\
\hline All patients & $10.1 \pm 1.7$ & $11.2 \pm 1.1$ & $<0.001$ \\
\hline Baseline anemia ( $n=92)$ & $10.0 \pm 1.6$ & $11.2 \pm 1.0$ & $<0.001$ \\
\hline eGFR pre-transplant & $9.0 \pm 3.7$ & & \\
\hline Post-operative day 1 & & $21.1 \pm 11.0$ & $<0.001$ \\
\hline Post-operative day 2 & & $53.1 \pm 27.1$ & $<0.001$ \\
\hline Post-operative day 7 & & $64.1 \pm 28.8$ & $<0.001$ \\
\hline Post-operative $1 \mathrm{yr}$ & & $61.7 \pm 20.9$ & $<0.001$ \\
\hline Mortality & & $2(2)$ & \\
\hline $\begin{array}{l}\text { Major adverse } \\
\text { cardiovascular event }\end{array}$ & & $14(14)$ & \\
\hline Graft failure & & $10(10)$ & \\
\hline Acute rejection & & $20(21)$ & \\
\hline
\end{tabular}

LVEF, left ventricular ejection fraction; $L V$, left ventricular; $L A$, left atrial; $R V$, right ventricular; eGFR, estimated glomerular filtration rate; $\mathrm{ACE}$, angiotensinconverting enzyme; ARB, angiotensin II receptor blocker.

Data are presented as mean \pm standard deviation or $\mathrm{n}(\%)$.

ed with improved LV diastolic function, evidenced by decreased E/e' ratio (Table 3). Moreover, eGFR at 1 year post-operation continued to be an independent predictor of improved LV diastolic function, by means of changes in E/e' ratio, after KT in multivariate analysis. Among patients with baseline diastolic dysfunction, ${ }^{17}$ the amount of fluid administered during surgery was an independent predictor of deterioration of diastolic function in terms of increase in E/e' ratio after transplantation.

\section{DISCUSSION}

In this single-center retrospective analysis of a consecutive cohort of patients with ESRD who underwent KT, we showed the significance of post-transplantation renal function recovery on improvement of $\mathrm{LV}$ diastolic function in terms of diminution of $\mathrm{E} / \mathrm{e}^{\prime}$ ratio. Moreover, the amount of administered intraoperative fluid was independently associated with deterioration of $\mathrm{LV}$ diastolic function, elucidating the importance of intraoperative fluid management among patients with pre-existing diastolic dysfunction.

The gold standard for assessing diastolic function is measuring the mean pulmonary capillary wedge pressure and LV end-diastolic pressure using cardiac catheterization. However, the invasiveness of this procedure deters it from being routinely applied to patients. Non-invasive Doppler echocardiographic parameters, such as E/e' ratio and LAVI, have also been used to estimate the LV filling pressure,$^{19}$ showing both reliability and validity for assessing diastolic function in patients with chronic kidney disease undergoing dialysis, as well as in the general population. ${ }^{20}$ Additionally, E/e' ratio has been shown to be a better reflection of the mean LV end-diastolic pressure, a surrogate for mean LA pressure, compared to other Doppler parameters. ${ }^{21}$ This study focused on the significance of $\mathrm{E} / \mathrm{e}^{\prime}$ ratio as a representative parameter of diastolic function and determined the relevant clinical and laboratory factors, which are known to influence diastolic function, that lead to proper patient management in patients with ESRD undergoing KT.

Patients with chronic kidney disease show characteristic traits of cardiac conditions, such as left ventricular hypertrophy and systolic and diastolic dysfunction, all of which converge into the diagnosis of uremic cardiomyopathy. Such changes in the cardiovascular system result from physiological responses to pressure and volume overload, underlying electrolyte imbalance, abnormal endocrinological conditions, and hemodynamic alterations. Among the various cardiovascular deteriorations, diastolic dysfunction is known to be a risk factor for postoperative morbidity, and even worse, post-operative survival in patients undergoing surgery. Such patients need more appropriate anesthetic management in order to prevent post-operative complications. Despite this significance, diastolic dysfunction is still overlooked in cardiac risk evaluation, because it is not usually a significant problem in the daily life of patients who do not suffer from exercise intolerance, dyspnea, or pulmonary edema. ${ }^{22}$ The American Heart Association-American College of Cardiology guidelines ${ }^{23}$ indicate that diastolic dysfunction is still underestimated in pre-operative risk assessment. In a previous study, pre-operative diastolic function showed predictive power for post-operative outcomes in patients undergoing KT. ${ }^{11}$ Therefore, this retrospective cohort study of patients undergoing KT was conducted to identify the perioperative clinical and laboratory factors related to aggravation of diastolic dysfunction, thereby emphasizing the need for ideal periop- 
Table 3. Analysis of Univariate and Multivariate Linear Regression: Predictors of Changes in Left Ventricular Diastolic Function after Kidney Transplantation

\begin{tabular}{|c|c|c|c|c|c|c|}
\hline & \multicolumn{4}{|c|}{$\begin{array}{c}\Delta E / e^{\prime} \text { ratio } \\
\text { (all patients, } n=97 \text { ) }\end{array}$} & \multicolumn{2}{|c|}{$\begin{array}{c}\Delta E / e^{\prime} \text { ratio } \\
\text { (patients with diastolic dysfunction, } \\
n=20)\end{array}$} \\
\hline & $\begin{array}{c}\text { Univariate coefficient } \\
(95 \% \mathrm{Cl})\end{array}$ & $p$ value & $\begin{array}{l}\text { Multivariate coefficient } \\
(95 \% \mathrm{CI})\end{array}$ & $p$ value & $\begin{array}{l}\text { Univariate coefficient } \\
\qquad(95 \% \mathrm{CI})\end{array}$ & $p$ value \\
\hline Transplant age & $0.005(-0.078$ to 0.089$)$ & 0.898 & & & $0.169(-0.149$ to 0.488$)$ & 0.278 \\
\hline Body mass index & $0.192(-0.116$ to 0.501$)$ & 0.219 & & & $-0.057(-0.741$ to 0.626$)$ & 0.862 \\
\hline Male & $-0.063(-1.936$ to 1.811$)$ & 0.947 & & & $-1.128(-6.067$ to 3.811$)$ & 0.637 \\
\hline Dialysis duration & $0.015(-0.008$ to 0.039$)$ & 0.193 & & & 0.029 (-0.037 to 0.095) & 0.367 \\
\hline$\Delta$ Mean blood pressure & $0.024(-0.030$ to 0.078$)$ & 0.378 & & & $0.077(-0.142$ to 0.296$)$ & 0.470 \\
\hline$\triangle \mathrm{eGFR}$, post-operative day 1 & $0.065(-0.025$ to 0.155$)$ & 0.154 & & & $0.195(-0.074$ to 0.463$)$ & 0.142 \\
\hline$\triangle \mathrm{eGFR}$, post-operative day 2 & $-0.011(-0.046$ to 0.023$)$ & 0.510 & & & $-0.007(-0.164$ to 0.151$)$ & 0.931 \\
\hline$\triangle \mathrm{eGFR}$, post-operative day 7 & $-0.033(-0.065$ to -0.002$)$ & 0.040 & $-0.011(-0.047-0.025)$ & 0.537 & $-0.055(-0.150$ to 0.041$)$ & 0.247 \\
\hline$\triangle \mathrm{eGFR}$, post-operative $1 \mathrm{yr}$ & $-0.063(-0.105$ to -0.021$)$ & 0.004 & $-0.056(-0.104-0.007)$ & 0.026 & $-0.054(-0.186$ to 0.078$)$ & 0.398 \\
\hline$\Delta$ Hemoglobin & $-0.418(-0.888$ to -0.053$)$ & 0.081 & & & $-1.048(-2.332$ to 0.237$)$ & 0.104 \\
\hline \multicolumn{7}{|l|}{ Intraoperative Input/output } \\
\hline Fluid & $0.000(-0.001$ to 0.001$)$ & 0.964 & & & 0.003 (0.000 to 0.005) & 0.029 \\
\hline Transfusion & $0.000(-0.003$ to 0.002$)$ & 0.746 & & & $0(-0.006$ to 0.006$)$ & 0.968 \\
\hline Urine Output & $0.001(-0.001$ to 0.003$)$ & 0.228 & & & $0.002(-0.003$ to 0.007$)$ & 0.432 \\
\hline Blood loss & $-0.003(-0.006$ to 0.000$)$ & 0.072 & & & $0(-0.008$ to 0.008$)$ & 0.954 \\
\hline Fluid, post-operative day 1 & $0(-0.001$ to 0.000$)$ & 0.121 & & & $-0.001(-0.001$ to 0.000$)$ & 0.201 \\
\hline Fluid, post-operative day 2 & $0(-0.001$ to 0.000$)$ & 0.065 & & & $-0.001(-0.002$ to 0.000$)$ & 0.208 \\
\hline Fluid, post-operative day 7 & $0(-0.001$ to 0.001$)$ & 0.691 & & & $-0.002(-0.004$ to 0.001$)$ & 0.118 \\
\hline Operation time & $-0.010(-0.025$ to 0.004$)$ & 0.169 & & & 0.032 (-0.025 to 0.089$)$ & 0.253 \\
\hline
\end{tabular}

$\mathrm{Cl}$, confidence interval; eGFR, estimated glomerular filtration rate.

erative management of patients with ESRD during KT. Patients with pre-existing LV systolic dysfunction were excluded to rule out the effects of systolic function on clinical outcomes.

In this study, we observed a significant relationship between the restoration of renal function and cardiac reverse remodeling after KT in terms of LV mass, LV end-diastolic dimension, LA volume, right ventricular systolic pressure, and E/e' ratio. Nevertheless, the majority of patients with ESRD, who are likely to suffer from pre-existing LV diastolic dysfunction (e.g., elevated $\mathrm{E} / \mathrm{e}^{\prime}$ ratio), exhibit different reactions in diastolic function, compared to other individuals with normal cardiac function during the perioperative period. Therefore, when discretely analyzed, the amount of fluid administered intraoperatively was identified to be associated with the changes in diastolic function after surgery. In other words, LV diastolic function improved as patient renal function recovered after transplantation, but deteriorated when given a greater amount of fluid during surgery, especially in patients with underlying diastolic dysfunction. Such findings are consistent with previous studies stating the impact of renal function and hemodynamic volume status on LV diastolic function in patients with ESRD. ${ }^{22,24,25}$ Our findings also coincide with the vulnerability of diastolic dysfunction on massive and acute fluid challenge during transplantation surgery, which is the type of surgery that is accompanied by extensive amounts of fluid administration over a relatively short period, and consequently aggravates diastolic function due to inadequate compensation.

In pursuit of understanding the significance of fluid management strategy during the post-operative period, a secondary statistical analysis of the relationship between changes in E/e' ratio and the amount of fluid administered during the post-operative period was conducted. As a result, there was no significant impact of the amount of fluid given at post-operative days 1,2 , and 7 on alterations in cardiac function in terms of changes in measured E/e' ratio (Table 3 ). However, our study succeeded in showing the significance of both intraoperative volume management and the vulnerability of the cardiovascular system on volume changes in patients with ESRD undergoing KT, which consequently clarified the specific efforts needed for such patients during renal transplantation surgery in an effort to protect perioperative cardiovascular function.

Our study had several limitations. First, this was a singlecenter retrospective study evaluating a small number of patients. Specifically, we investigated evaluable patients who survived during the perioperative period to allow both pre- and post-operative TTE, and the possibility of selection bias might have influenced the results. Second, different time intervals for follow-up TTEs need to be considered. Echocardiograms were done at different time intervals after surgery based upon clinical need, instead of being performed at designated times. 
This limited our ability to properly evaluate changes in diastolic function over a fixed period. Thus, further studies using regular follow-up post-operative TTEs should be considered for more detailed interpretations on changes in diastolic function after KT in patients with ESRD. Moreover, as some of our enrolled patients had been scheduled for preemptive KT before full-blown kidney failure, such patient characteristics may have affected the perioperative outcomes, including cardiac functions. Although our results indicated a significant relationship reflective of how intraoperative LV loading condition can affect postoperative diastolic performance, more extensive and in-depth study is needed for thorough explanation of the influence of occult volume overload in KT, altered physiology of vascular endothelium and degraded diastolic function of heart. Lastly, the inherent limitations in echocardiographic methods used to quantify diastolic function need to be considered. However, although other means of assessment, such as cardiac magnetic resonance, are considered as the "gold standard" for evaluating cardiac structure and function, echocardiography remains an important clinical and research tool when considering its availability and practicality.

In conclusion, this study reported a significant relationship between post-operative renal function and changes in LV diastolic function after renal transplantation surgery in patients with ESRD. Additionally, the significance of intraoperative fluid management on changes in LV diastolic function, especially in patients with pre-existing diastolic dysfunction prior to surgery, was observed. Despite the limitations, this study succeeded in identifying the important transition of LV diastolic function of patients with ESRD after KT and also supported the establishment of definite guidelines for intra- and post-operative management plans for such patients. LV diastolic dysfunction is an independent risk factor for post-operative major adverse cardiovascular events and mortality after transplantation. Therefore, optimal intraoperative fluid management may reduce the risk of worsening diastolic dysfunction and improve post-KT renal function in patients with pretexting diastolic dysfunction.

\section{ACKNOWLEDGEMENTS}

This study was supported by a new faculty research seed money grant of Yonsei University College of Medicine for 2017 (201732-0027).

\section{AUTHOR CONTRIBUTIONS}

Conceptualization: Eun Jung Kim, So Yeon Kim, and Yong Seon Choi. Data curation: Eun Jung Kim, So Yeon Kim, and Soojeong Kang. Formal analysis: Eun Jung Kim, Soojeong Kang, and Yong Seon Choi. Funding acquisition: Eun Jung Kim. Investigation: Eun Jung Kim, Bon-Nyeo Koo, Kyu Ha Huh, and Yong Seon Choi. Methodology: Eun Jung Kim and Yong Seon Choi. Project administration: Eun Jung Kim, Bon-Nyeo Koo, Kyu Ha Huh, and Yong Seon Choi. Resources: Eun
Jung Kim, Kyu Ha Huh, Soojeong Kang, and Yong Seon Choi. Software: Eun Jung Kim and Yong Seon Choi. Supervision: Bon-Nyeo Koo and Yong Seon Choi. Validation: Bon-Nyeo Koo and Yong Seon Choi. Visualization: Eun Jung Kim. Writing-original draft: Eun Jung Kim. Writing_review \& editing: Bon-Nyeo Koo, So Yeon Kim, Kyu Ha Huh, Soojeong Kang, and Yong Seon Choi.

\section{ORCID iDs}

Eun Jung Kim

Bon-Nyeo Koo

So Yeon Kim

Kyu Ha Huh

Soojeong Kang

Yong Seon Choi

http://orcid.org/0000-0002-5693-1336

https://orcid.org/0000-0002-3189-1673 https://orcid.org/0000-0001-5352-157X https://orcid.org/0000-0003-1364-6989 https://orcid.org/0000-0003-1778-4457 https://orcid.org/0000-0002-5348-864X

\section{REFERENCES}

1. Block GA, Klassen PS, Lazarus JM, Ofsthun N, Lowrie EG, Chertow GM. Mineral metabolism, mortality, and morbidity in maintenance hemodialysis. J Am Soc Nephrol 2004;15:2208-18.

2. Pecoits-Filho R, Bucharles S, Barberato SH. Diastolic heart failure in dialysis patients: mechanisms, diagnostic approach, and treatment. Semin Dial 2012;25:35-41.

3. Losi MA, Memoli B, Contaldi C, Barbati G, Del Prete M, Betocchi S, et al. Myocardial fibrosis and diastolic dysfunction in patients on chronic haemodialysis. Nephrol Dial Transplant 2010;25:1950-4.

4. Glassock RJ, Pecoits-Filho R, Barberato SH. Left ventricular mass in chronic kidney disease and ESRD. Clin J Am Soc Nephrol 2009;4 Suppl 1:S79-91.

5. Hawwa N, Shrestha K, Hammadah M, Yeo PSD, Fatica R, Tang WHW. Reverse remodeling and prognosis following kidney transplantation in contemporary patients with cardiac dysfunction. J Am Coll Cardiol 2015;66:1779-87.

6. Ferreira SR, Moisés VA, Tavares A, Pacheco-Silva A. Cardiovascular effects of successful renal transplantation: a 1-year sequential study of left ventricular morphology and function, and 24-hour blood pressure profile. Transplantation 2002;74:1580-7.

7. Hewing B, Dehn AM, Staeck O, Knebel F, Spethmann S, Stangl K, et al. Improved left ventricular structure and function after successful kidney transplantation. Kidney Blood Press Res 2016;41:701-9.

8. Wali RK, Wang GS, Gottlieb SS, Bellumkonda L, Hansalia R, Ramos E, et al. Effect of kidney transplantation on left ventricular systolic dysfunction and congestive heart failure in patients with end-stage renal disease. J Am Coll Cardiol 2005;45:1051-60.

9. Sharma R, Pellerin D, Gaze DC, Mehta RL, Gregson H, Streather CP, et al. Mitral peak Doppler E-wave to peak mitral annulus velocity ratio is an accurate estimate of left ventricular filling pressure and predicts mortality in end-stage renal disease. J Am Soc Echocardiogr 2006;19:266-73.

10. Higashi M, Yamaura K, Ikeda M, Shimauchi T, Saiki H, Hoka S. Diastolic dysfunction of the left ventricle is associated with pulmonary edema after renal transplantation. Acta Anaesthesiol Scand 2013; 57:1154-60.

11. Kim EJ, Chang S, Kim SY, Huh KH, Kang S, Choi YS. Predictive value of echocardiographic abnormalities and the impact of diastolic dysfunction on in-hospital major cardiovascular complications after living donor kidney transplantation. Int J Med Sci 2016;13:620-8.

12. El-Husseini AA, Sheashaa HA, Hassan NA, El-Demerdash FM, Sobh MA, Ghoneim MA. Echocardiographic changes and risk factors for left ventricular hypertrophy in children and adolescents after renal transplantation. Pediatr Transplant 2004;8:249-54. 
13. Levey AS, Bosch JP, Lewis JB, Greene T, Rogers N, Roth D. A more accurate method to estimate glomerular filtration rate from serum creatinine: a new prediction equation. Modification of Diet in Renal Disease Study Group. Ann Intern Med 1999;130:461-70.

14. Kidney Disease: Improving Global Outcomes (KDIGO) Anemia Work Group. KDIGO Clinical Practice Guideline for anemia in chronic kidney disease. Kidney Int Suppl 2012;2:279-335.

15. Sohn DW, Chai IH, Lee DJ, Kim HC, Kim HS, Oh BH, et al. Assessment of mitral annulus velocity by Doppler tissue imaging in the evaluation of left ventricular diastolic function. J Am Coll Cardiol 1997;30:474-80.

16. Kim YJ, Sohn DW. Mitral annulus velocity in the estimation of left ventricular filling pressure: prospective study in 200 patients. J Am Soc Echocardiogr 2000;13:980-5.

17. Nagueh SF, Smiseth OA, Appleton CP, Byrd BF 3rd, Dokainish H, Edvardsen T, et al. Recommendations for the evaluation of left ventricular diastolic function by echocardiography: an update from the American Society of Echocardiography and the European Association of Cardiovascular Imaging. J Am Soc Echocardiogr 2016;29:277-314.

18. Lang RM, Badano LP, Mor-Avi V, Afilalo J, Armstrong A, Ernande L, et al. Recommendations for cardiac chamber quantification by echocardiography in adults: an update from the American Society of Echocardiography and the European Association of Cardiovascular Imaging. J Am Soc Echocardiogr 2015;28:1-39.

19. Nagueh SF, Middleton KJ, Kopelen HA, Zoghbi WA, Quiñones MA. Doppler tissue imaging: a noninvasive technique for evaluation of left ventricular relaxation and estimation of filling pressures. J Am
Coll Cardiol 1997;30:1527-33.

20. Ommen SR, Nishimura RA, Appleton CP, Miller FA, Oh JK, Redfield MM, et al. Clinical utility of Doppler echocardiography and tissue Doppler imaging in the estimation of left ventricular filling pressures: a comparative simultaneous Doppler-catheterization study. Circulation 2000;102:1788-94.

21. Hayashi SY, Rohani M, Lindholm B, Brodin LA, Lind B, Barany P, et al. Left ventricular function in patients with chronic kidney disease evaluated by colour tissue Doppler velocity imaging. Nephrol Dial Transplant 2006;21:125-32.

22. Ryu T, Song SY. Perioperative management of left ventricular diastolic dysfunction and heart failure: an anesthesiologist's perspective. Korean J Anesthesiol 2017;70:3-12.

23. Fleisher LA, Fleischmann KE, Auerbach AD, Barnason SA, Beckman JA, Bozkurt B, et al. 2014 ACC/AHA guideline on perioperative cardiovascular evaluation and management of patients undergoing noncardiac surgery: a report of the American College of Cardiology/American Heart Association Task Force on practice guidelines. J Am Coll Cardiol 2014;64:e77-137.

24. Ybarra J, Sánchez-Hernández J, Vilallonga R, Romeo JH. Differential associations between glomerular filtration rate and duration of obesity depending on the presence or absence of left ventricular diastolic dysfunction. Eur J Intern Med 2016;32:47-52.

25. Calleja AM, Rakowski H, Williams LK, Jamorski M, Chan CT, Carasso S. Left atrial and ventricular systolic and diastolic myocardial mechanics in patients with end-stage renal disease. Echocardiography 2016;33:1495-503. 